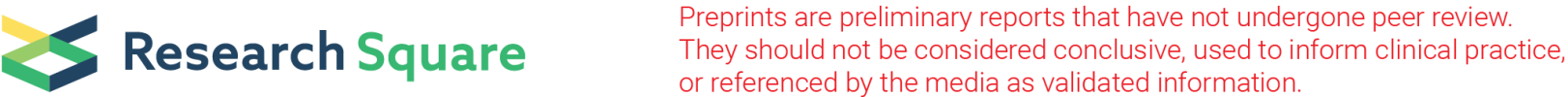

\section{Interrelationship Between 2019-nCov Receptor DPP4 and Diabetes Mellitus Targets Based on Protein Interaction Network}

Qian Gao ( $\sim 220293991 @ q q . c o m)$

Affiliated Hospital of Shaoxing University of Edocrine

\section{Wenjun Zhang}

Affiliated Hospital of Shaoxing University of Edocrine

Tingting Li

Affiliated Hospital of Shaoxing University of Edocrine

Guojun Yang

Affiliated Hospital of Shaoxing University of Edocrine

Wei Zhu

Affiliated Hospital of Shaoxing University of Edocrine

Naijun Chen

Affiliated Hospital of Shaoxing University of Edocrine

Huawei Jin

Affiliated Hospital of Shaoxing University of Edocrine

\section{Research Article}

Keywords: COVID-19, Diabetes mellitus, Protein interaction network, DPP4

Posted Date: June 29th, 2021

DOl: https://doi.org/10.21203/rs.3.rs-653800/v1

License: (c) (i) This work is licensed under a Creative Commons Attribution 4.0 International License.

Read Full License

Version of Record: A version of this preprint was published at Scientific Reports on January 7th, 2022.

See the published version at https://doi.org/10.1038/s41598-021-03912-6. 


\section{Abstract}

Objective: Exploring the relationship between diabetes mellitus targets and DPP4 of the receptor of novel coronavirus (2019-nCoV) through a protein interaction network to provide new perspective for clinical medication.

Methods: Diabetes mellitus targets were obtained from GeneCards database. Targets with a relevance score exceeding 20 were included, and DPP4 protein was added manually. The initial protein interaction network was obtained through String. The targets directly related to DPP4 were selected as the final analysis targets. Importing them into String again to obtain the protein interaction network. Module identification, GO analysis and KEGG pathway analysis were carried out respectively. The impact of DPP4 on the whole network was analyzed by scoring the module where it located.

Results: 43 DPP4-related proteins were finally selected from the diabetes mellitus targets and three functional modules were found by the cluster analysis. Module 1 was involved in insulin secretion and glucagon signaling pathway, module 2 and module 3 were involved in signaling receptor binding. The scoring results showed that LEP and apoB in module 1 were the highest, and the scores of INS, IL6 and ALB of cross module associated proteins of module 1 were the highest.

Conclusions: DPP4 is widely associated with key proteins in diabetes mellitus. COVID-19 may affect DPP4 in patients with diabetes mellitus, leading to high mortality of diabetes mellitus combined with COVID-19. DPP4 inhibitors and IL-6 antagonists can be considered to reduce the effect of COVID-19 infection on diabetic patients.

\section{Introduction}

Due to the high prevalence and long incubation periods often without symptoms, the severe acute respiratory syndrome coronavirus-2 (SARS-CoV-2) has infected hundreds of millions of people around the world, causing the coronavirus disease 2019 (COVID-19) pandemic. ${ }^{[1]}$. Recent studies have shown that DPP-4 is one of the functional receptors of human coronavirus. ${ }^{[2-5]}$ SARS-CoV-2 Virus S protein can infect the body by acting on this receptor of bronchiolar epithelial cells. ${ }^{[6]}$ Dipeptidyl peptidase-4(DPP4) also known as CD26, is a serine exopeptidase, a multifunctional type-II transmembrane glycoprotein that presents in a dimeric form on the cell surface. DPP4 is multifunctional, highly conserved among mammals. It regulate the activities of peptide hormones, neuropeptides, cytokines and growth factors, but also act as a surface antigen to cooperate with other molecules or proteins to mediate the interaction between cells and matrix, cells and cells, and play various regulatory roles in immune activation, inflammatory response and tumorigenesis. ${ }^{[7]}$

DPP4 inhibitors are a kind of hypoglycemic drugs which have been widely used. DPP4 can decompose glucagon like peptide-1 (GLP-1), which can stimulate insulin secretion and DPP4 inhibitors can effectively antagonize this effect. They can control blood glucose, especially postprandial blood glucose, and 
improve glucose tolerance, insulin resistance and other symptoms of patients by inhibiting the degradation of GLP-1. ${ }^{[8]}$ Diabetic patients are more likely to be infected with COVID-19, and the risk of death is significantly higher than ordinary patients. ${ }^{\left[{ }^{9-10]}\right.}$ Current studies have shown that DPP4 inhibitors can be considered as the preferred hypoglycemic regimen in the treatment of diabetic patients with COVID-19 infection. ${ }^{[11]}$ In order to strengthen the management of diabetic patients with COVID-19, we intend to study the protein interaction through the protein interaction network, and evaluate the degree of protein-protein correlation through the correlation score. Based on the String database, this paper analyzes the relationship between DPP4 and diabetic protein targets, in order to find a new clue for the management of diabetic patients with COVID-19.

\section{Materials And Methods}

\subsection{Get diabetes mellitus targets information}

All targets of diabetes mellitus were obtained through GeneCards database (https://www.genecards.org/). Targets with a relevance score exceeding 20 were included, and DPP4 protein was added manually as target point.

\subsection{Screening of protein targets directly associated with DPP4}

The target was imported into the STRING (https://string-db.org/) ${ }^{[12]}$ in the form of symbol to obtain the initial protein network, and the targets directly related to DPP4 was screened.

\subsection{Construction of DPP4 related protein network}

DPP4 and 43 related proteins were re-imported into STRING to obtain the protein interaction network for subsequent analysis.

\subsection{Identification and analysis of DPP4 related protein network modules}

K-means algorithm was used to cluster the above protein interaction networks.

\subsection{Gene ontology(GO)and Kyoto encyclopedia of genes and genomes $\square$ KEGG $\square$ pathway analysis of each module}

GO analysis and KEGG pathway analysis of all modules are further carried out through STRING.

2.6. Evaluation of the impact of DPP4 on the whole protein network 
In order to further evaluate the impact of DPP4 on the whole network and the possible pathway, the module 1 where DPP4 is located is used as the internal network, the cross module association of internal network is screened out. The statistical description and mapping are carried out. The data are filtered with 0.6 (determined by mean and median) as the standard, the qualified cross module associated proteins and associated scores were listed, and the proteins included in module 1 and the cross module associated protein scores of module 1 were summed as the scores.

\section{Results}

\subsection{Screening results of DPP4 associated proteins}

A total of 1031 diabetes mellitus targets and 43 targets directly related to DPP4 were obtained by screening (Table 1). 
Table 1

DPP4 related protein

\begin{tabular}{|c|c|c|}
\hline Symbol & Protein Name & Score \\
\hline ACE & Angiotensin-converting enzyme & 0.709 \\
\hline ADIPOQ & Adiponectin & 0.572 \\
\hline AKT1 & RAC-alpha serine/threonine-protein kinase & 0.52 \\
\hline ALB & Serum albumin & 0.671 \\
\hline APOB & Apolipoprotein B-100 & 0.438 \\
\hline APOE & Apolipoprotein E & 0.422 \\
\hline CAV1 & Caveolin-2 & 0.692 \\
\hline CCL2 & C-C motif chemokine 2 & 0.448 \\
\hline CCR5 & C-C chemokine receptor type 5 & 0.4 \\
\hline CPE & Carboxypeptidase E & 0.438 \\
\hline CRP & C-reactive protein & 0.536 \\
\hline CTLA4 & Cytotoxic T-lymphocyte protein 4 & 0.44 \\
\hline CXCL10 & C-X-C motif chemokine 10 & 0.581 \\
\hline FN1 & Fibronectin type III domain containing & 0.773 \\
\hline GCG & Glucagon & 0.994 \\
\hline GCGR & Glucagon receptor & 0.573 \\
\hline GCK & Glucokinase & 0.461 \\
\hline GGT1 & Glutathione hydrolase 1 proenzyme & 0.411 \\
\hline GHRL & Appetite-regulating hormone & 0.559 \\
\hline GLP1R & Glucagon-like peptide 1 receptor & 0.899 \\
\hline GPT & Alanine aminotransferase 1 & 0.508 \\
\hline HNF1A & Hepatocyte nuclear factor 1-alpha & 0.538 \\
\hline IAPP & Islet amyloid polypeptide & 0.672 \\
\hline ICAM1 & Intercellular adhesion molecule 1 & 0.441 \\
\hline IL10 & Interleukin-10 & 0.41 \\
\hline IL6 & Interleukin-6 & 0.514 \\
\hline INS & Insulin & 0.942 \\
\hline
\end{tabular}




\begin{tabular}{|lll|}
\hline Symbol & Protein Name & Score \\
\hline INS-IGF2 & Insulin, isoform 2 & 0.535 \\
\hline LEP & Leptin & 0.593 \\
\hline MMP2 & 72 kDa type IV collagenase & 0.404 \\
\hline NOS3 & Matrix metalloproteinase-9 & 0.405 \\
\hline NPY & Pro-neuropeptic oxide synthase, endothelial & 0.658 \\
\hline PPARG & Peroxisome proliferator-activated receptor gamma & 0.735 \\
\hline REN & Renin & 0.51 \\
\hline SERPINE1 & Plasminogen activator inhibitor 1 & 0.499 \\
\hline SLC2A2 & Solute carrier family 2, facilitated glucose transporter member 2 & 0.462 \\
\hline SLC2A4 & Solute carrier family 2, facilitated glucose transporter member 4 & 0.461 \\
\hline SLC5A2 & Sodium/glucose cotransporter 2 & 0.892 \\
\hline SST & Somatostatin & 0.472 \\
\hline TNF & Tumor necrosis factor & 0.473 \\
\hline VCAM1 & Vascular cell adhesion protein 1 & 0.416 \\
\hline VEGFA & Vascular endothelial growth factor A & 0.643 \\
\hline
\end{tabular}

\subsection{Protein interaction network results}

The network consists of 44 nodes and 570 edges. The average value of nodes is 25.9. (Fig. 1).

\subsection{Cluster analysis results of protein interaction network}

Three functional modules were obtained by cluster analysis. Module 1 includes 17 nodes (SLC5A2 SLC2A2ロLEP $\square G C G \square D P P 4 \square A D I P O Q \square A P O B \square G G T 1 \square G P T \square N P Y \square G C K \square C P E \triangle S S T \square G C G R \square I A P P \square G L P 1 R \square G H R L) ;$

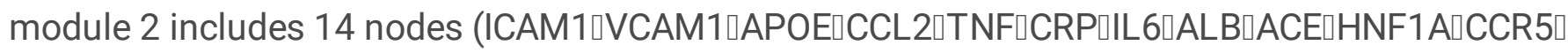

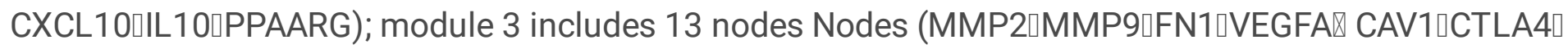

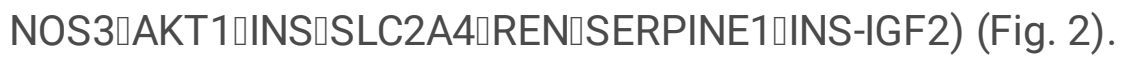

\subsection{GO analysis and KEGG analysis}

Through GO analysis and KEGG analysis, it was found that the target of module 1 was mainly enriched in insulin secretion and glucagon signal transduction pathway; the target of module 2 and module 3 was mainly enriched in signaling receptor binding. 


\subsection{Module 1 cross module correlation statistics results}

Module 1 contains 190 cross module associations, accounting for $33 \%$ of the total number of network associations (570). The average score of association is 0.649 , and the median is 0.628 (Table 2).

Table 2

GO and KEGG analysis of different modules

\begin{tabular}{|c|c|c|c|}
\hline Module & Protein & $\begin{array}{l}\text { GO and KEGG } \\
\text { analysis }\end{array}$ & $P$ \\
\hline \multirow[t]{6}{*}{$\begin{array}{l}\text { Module } \\
1\end{array}$} & \multirow[t]{6}{*}{ 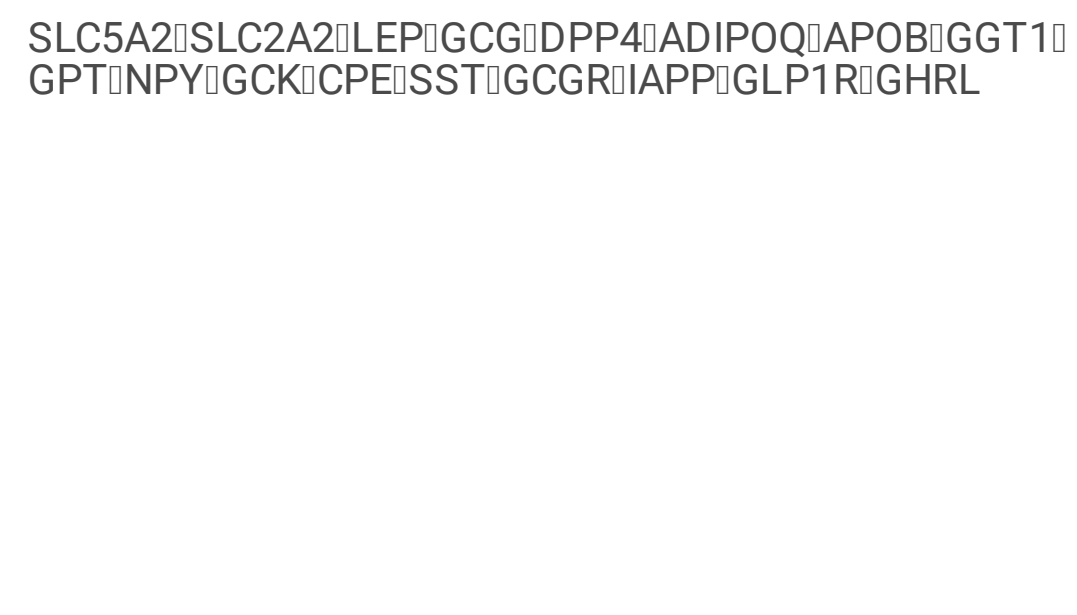 } & & $\begin{array}{l}1.29 \mathrm{e}- \\
07\end{array}$ \\
\hline & & hormone activity & \\
\hline & & KEGG: & 05 \\
\hline & & $\begin{array}{l}\text { Maturity onset } \\
\text { diabetes of the } \\
\text { young; }\end{array}$ & $\begin{array}{l}3.55 \mathrm{e}- \\
05 \\
3.55 \mathrm{e}- \\
05\end{array}$ \\
\hline & & Insulin secretion; & \\
\hline & & $\begin{array}{l}\text { Glucagon } \\
\text { signaling pathway }\end{array}$ & \\
\hline \multirow[t]{5}{*}{$\begin{array}{l}\text { Module } \\
2\end{array}$} & \multirow[t]{5}{*}{ 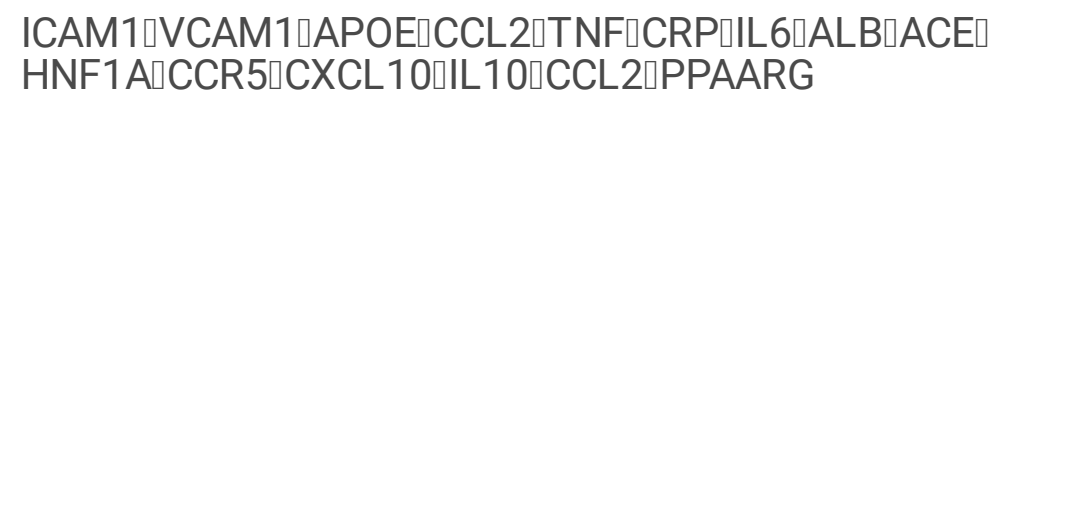 } & & $\begin{array}{l}2.63 e- \\
05\end{array}$ \\
\hline & & binding & $\begin{array}{l}9.61 \mathrm{e}- \\
08\end{array}$ \\
\hline & & KEGG: & \\
\hline & & $\begin{array}{l}\text { African } \\
\text { trypanosomiasis; }\end{array}$ & 08 \\
\hline & & $\begin{array}{l}\text { TNF signaling } \\
\text { pathway }\end{array}$ & \\
\hline \multirow{4}{*}{$\begin{array}{l}\text { Module } \\
3\end{array}$} & \multirow{4}{*}{ 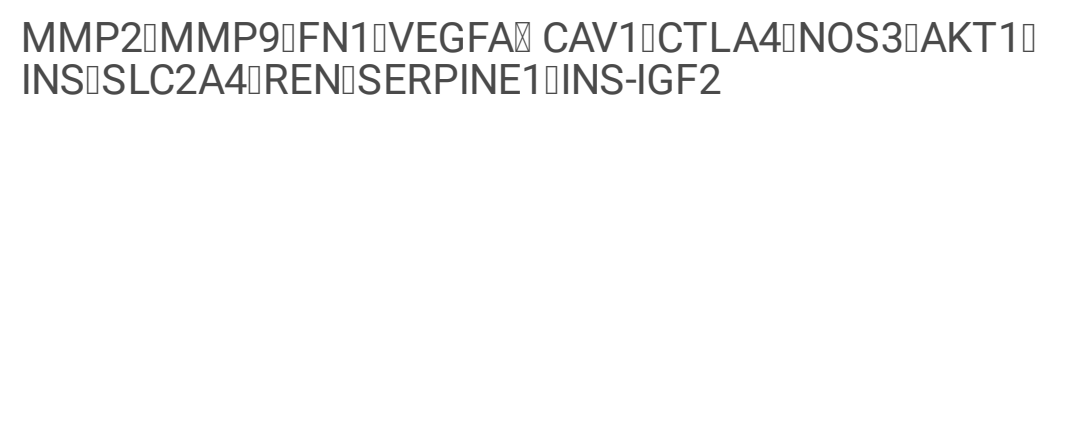 } & GO: & 0.0030 \\
\hline & & $\begin{array}{l}\text { signaling receptor } \\
\text { binding }\end{array}$ & $\begin{array}{l}2.14 \mathrm{e}- \\
08\end{array}$ \\
\hline & & KEGG: & \\
\hline & & $\begin{array}{l}\text { Fluid shear stress } \\
\text { and } \\
\text { atherosclerosis }\end{array}$ & \\
\hline
\end{tabular}

\subsection{Module 1 cross module correlation impact assessment results}


In module 1, LEP and apoB have the highest scores, which indicates that the above proteins may have cross module effects. The scores of INS, IL 6 and ALB of cross module associated proteins of module 1 were the highest, which indicates that the effects of module 1 on other modules are more likely to be achieved through interaction with the above proteins (Table 3 ). 
Table 3

Module 1 cross module associated protein and relevance score

\begin{tabular}{|c|c|c|c|c|c|c|c|c|c|}
\hline \multicolumn{2}{|l|}{ APOB } & \multicolumn{2}{|c|}{ GGT1 } & \multicolumn{2}{|l|}{ GPT } & \multicolumn{2}{|l|}{ NPY } & \multicolumn{2}{|l|}{ CPE } \\
\hline NOS3 & 0.707 & IL6 & 0.616 & IL10 & 0.66 & IL6 & 0.709 & INS & 0.97 \\
\hline VCAM1 & 0.74 & CRP & 0.669 & TNF & 0.707 & INS & 0.889 & & \\
\hline IL10 & 0.751 & INS & 0.67 & IL6 & 0.733 & CXCL10 & 0.908 & & \\
\hline MMP9 & 0.789 & ALB & 0.805 & INS & 0.839 & CCR5 & 0.919 & & \\
\hline TNF & 0.793 & & & CRP & 0.896 & & & & \\
\hline INS & 0.848 & & & ALB & 0.924 & & & & \\
\hline IL6 & 0.969 & & & & & & & & \\
\hline SST & & GCG & & GHRL & & GCG & & LEP & \\
\hline ALB & 0.621 & INS & 0.694 & INS-IGF2 & 0.607 & INS-IGF2 & 0.668 & REN & 0.662 \\
\hline AKT1 & 0.68 & & & NOS3 & 0.628 & SERPINE1 & 0.672 & CAV1 & 0.682 \\
\hline CXCL10 & 0.908 & & & IL6 & 0.63 & ALB & 0.704 & INS-IGF2 & 0.7 \\
\hline INS & 0.915 & & & ALB & 0.675 & AKT1 & 0.776 & MMP9 & 0.707 \\
\hline \multirow[t]{15}{*}{ CCR5 } & 0.922 & & & AKT1 & 0.71 & SLC2A4 & 0.793 & VCAM1 & 0.722 \\
\hline & & & & INS & 0.944 & INS & 0.986 & MMP2 & 0.724 \\
\hline & & & & & & & & CCL2 & 0.732 \\
\hline & & & & & & & & ICAM1 & 0.762 \\
\hline & & & & & & & & NOS3 & 0.762 \\
\hline & & & & & & & & APOE & 0.775 \\
\hline & & & & & & & & IL10 & 0.797 \\
\hline & & & & & & & & ALB & 0.803 \\
\hline & & & & & & & & AKT1 & 0.841 \\
\hline & & & & & & & & SLC2A4 & 0.856 \\
\hline & & & & & & & & VEGFA & 0.867 \\
\hline & & & & & & & & SERPINE1 & 0.879 \\
\hline & & & & & & & & TNF & 0.9 \\
\hline & & & & & & & & IL6 & 0.943 \\
\hline & & & & & & & & INS & 0.976 \\
\hline
\end{tabular}




\begin{tabular}{|c|c|c|c|c|c|}
\hline APOB & GGT1 & GPT & NPY & CPE & \\
\hline & & & & CRP & 0.981 \\
\hline
\end{tabular}

\section{Discussion}

By sorting the correlation scores of DPP4 related proteins, we found that GCG, INS and GLP1R had the highest correlation scores, which were more than 0.8, and GCG and INS were more than 0.9, which meant that DPP4 was most closely related to the above diabetes related proteins. By cluster analysis, three functional modules were found. Module 1 was mainly involved in insulin secretion and glucagon signal transduction pathway including DPP4, while module 2 and module 3 were involved in signaling receptor binding. The binding of S protein of COVID-19 with DPP4 is the starting point of the course of the disease. In order to evaluate the possible influence and pathway of the virus after entering the human body, DPP4 is taken as the starting point of the whole network for in-depth analysis. Because there are a lot of low values in the correlation scores of DPP4 cross module associated proteins, the mean and median are used as the standard to filter the data, and the sum of the correlation scores is used as the score. It was found that LEP and apoB were the highest in module 1. The scores of INS, IL6 and ALB of cross module associated proteins of module 1 were the highest. It is worth noting that DPP4 cross module correlation scores are less than 0.6 , so it does not shown. Therefore, the abnormal changes of DPP4 may not play an effect by directly interacting with module associated proteins, but may be that module 1 magnifies its effect, and this effect is transmitted to module 2 and module 3 through the close relationship between module 1 and INS, IL6 and ALB, resulting in the disorder of glucose metabolism and inflammatory regulation.

The data of clinical trials related to COVID-19 show that diabetic patients are more likely to be infected with SAR-COV-2, while the prognosis of patients with diabetes mellitus is worse and the risk of death is higher. ${ }^{[9-10]}$ So, what is the mechanism of susceptibility to SAR-COV-2 in diabetic patients? What is the mechanism of higher risk of death and worse prognosis in patients with diabetes mellitus? How to guide the medication of diabetic patients infected with SAR-COV-2 in clinic? DPP4 as a type II transmembrane protein is also known to be cleaved from the cell membrane involving different metalloproteases in a celltype-specific manner. Circulating, soluble DPP4 has been identified as a new adipokine, which exerts both para- and endocrine effects. ${ }^{[13]}$ Recently, studies have found that SCD26 serum protein levels are reduced in diabetes. High serum SCD26 level could protect from viral infection by blocking the receptor from virus entry, whereas low SCD26 level may be associated with a higher risk of infection which may be one of the mechanisms of susceptibility to SARS-COV-2 in diabetic patients. ${ }^{[14]}$ The high mortality of patients with COVID-19 are closely related to the disorder of glucose metabolism and inflammation regulation. ${ }^{[15]}$ The $S$ protein of SARS-COV-2 can invade T, NK and other immune cells through binding receptor DPP4, and activate nuclear factor $-\mathrm{K} B(\mathrm{NF}-\mathrm{K} b)$ pathway, resulting in the secretion of a series of pro-inflammatory cytokines, including interleukin-6 (IL-6). ${ }^{[16]}$ IL-6 can promote the differentiation of Thelper cell 17 (Th17) and other lymphocyte changes. Circulating IL-6 and soluble IL-6 receptor complexes indirectly activate 
many types of cells, including endothelial cells, leading to the proliferation of a series of cytokines, leading to decreased blood pressure and acute respiratory distress syndrome (ARDS) ${ }^{[17]}$ IL-6 plays a key role in this cascade. It suggests that people should evaluate the possibility of IL- 6 antagonists (such as tocilizumab, sarilumab and siluximab) in the treatment of severe COVID-19 disease. DPP4 also degrades GLP-1 and GIP and plays an important role in glucose metabolism. Studies have shown that GLP-1-based therapy can reduce the activation of immune cells, inhibit the release of pro-inflammatory cytokines, and reduce organ dysfunction and mortality. ${ }^{[18]}$ DPP4 inhibitor can increase the half-life of GLP-1 and play an indirect role. DPP4 inhibitors can also resist lung inflammation and reduce lung injury. ${ }^{[19]}$ These studies suggest that DPP4 inhibitors may play an active role in the treatment of diabetic patients with COVID-19. However, the issue remains controversial. Males believes that DPP4 inhibitors inhibit the immune system and may increase the risk of infection. ${ }^{[20]}$ However,There are also studies that show that the use of DPP4 inhibitors does not have a negative impact. ${ }^{[21]}$ Previous retrospective studies have found that DPP4 inhibitors have serious heterogeneity in the treatment effect of COVID-19. ${ }^{[22-28]}$ However, these studies are not randomized controlled double-blind studies. At present, three randomized controlled trials (NCT 04341935区NCT 04371978ロNCT04365517)(Retrieved from: https://clinicaltrials.gov/) are ongoing to study the effect of DPP4 inhibitors on the prognosis of COVID-19 and the clinical results are expected to be obtained as soon as possible.

Leptin was the protein with the highest cross module effect in module 1, with a score of 16.071. Leptin is a hormone secreted by adipose tissue. When the body fat is reduced or in a low-energy state (such as starvation), leptin will decrease significantly, thus stimulating the food seeking behavior and reducing its own energy consumption. On the contrary, when the body fat increases, leptin increases, which inhibits eating and accelerates metabolism. ${ }^{[29]}$ Leptin not only regulates body weight, but also is associated with monocyte activation and severe illness in patients with COVID-19. ${ }^{[30]}$ Overweight patients with COVID-19 tend to have higher leptin levels, which further activates monocytes, leading to amplification or imbalance of immune response, which may also be the mechanism of overweight patients more prone to serious diseases. ${ }^{[31]}$ CRS is one of the important causes of death in patients with COVID - 19. However, the changes of cytokine profile and the underlying mechanism are still unknown. Using the cytokine array containing 174 cytokines related to inflammation found that the cytokine spectrum of severe patients with COVID-19 was significantly different from that of mild patients or healthy controls. Leptin, CXCL-10, IL-6, IL-10, IL-12, TNF and other cytokines, indicating that these inflammatory factors can predict the severity of COVID - 19 disease. ${ }^{[32]}$ The cluster analysis showed that leptin in module 1 and IL-6, IL-10, TNF in module 2 had a cross model effect, and the highest score of IL-6 was 6.163 , which further suggested the possibility of IL- 6 antagonists in the treatment of severe COVID-19 disease.

In this paper, we screened out the diabetes related proteins and functional modules closely related to DPP4 through protein interaction network, and analyzed the influence of DPP4 module 1 on the whole protein network and the possible pathway. It was proposed that the influence of COVID-19 infection was amplified by DPP4 in diabetic patients, and through its interaction with INS, leptin, IL-6 and other proteins. Increased glucose metabolism disorder and excessive inflammatory reaction lead to the high mortality in 
diabetic patients with COVID-19. At present, the data of retrospective observational studies show that the therapeutic effect of DPP4 inhibitors has serious heterogeneity, but the strength of the above studies is low. We look forward to further randomized controlled trials to verify our inference.

\section{Conclusions}

DPP4 is widely associated with key proteins in diabetes mellitus. COVID-19 may affect DPP4 in patients with diabetes mellitus, leading to high mortality of diabetes mellitus combined with COVID-19. DPP4 inhibitors and IL- 6 antagonists can be considered to reduce the effect of COVID-19 infection on diabetic patients.

\section{Abbreviations}

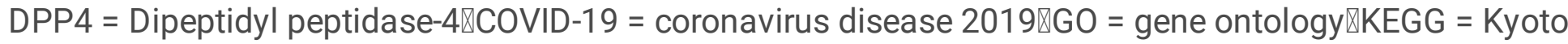
encyclopedia of genes and genomes $₫ \mathrm{GLP}-1$ = glucagon like peptide-1

\section{Declarations}

\section{Author contributions}

Conceptualization and design: Qian Gao and Wenjun Zhang.

Data curation $₫$ Qian Gao, Naijun Chen, Wenjun Zhang, Guojun Yang, Huawei Jin.

Formal analysis $\bigotimes$ Tingting Li.

Writing $₫ Q$ Qian Gao.

All authors read and approved the final manuscript.

\section{References}

1. JY JYCC, MN MNTT, YJ YJKK. COVID-19 vaccines: The status and perspectives in delivery points of view. Adv Drug Deliv Rev 2020;170:1-25.

2. GN GNBB, F FPP. Structural and functional modelling of SARS-CoV-2 entry in animal models. Sci Rep 2020;10(1):15917.

3. N NVV, JA JAWW. Emerging WuHan (COVID-19) coronavirus: glycan shield and structure prediction of spike glycoprotein and its interaction with human CD26. Emerg Microbes Infect 2020; 9(1):601-4.

4. Li Y, Zhang Z, Yang L, et al. The MERS-CoV Receptor DPP4 as a Candidate Binding Target of the SARS-CoV-2 Spike. iScience 2020; 23(6):101160.

5. U URR, M MDD, G GTT, et al. Distribution of ACE2, CD147, CD26, and other SARS-CoV-2 associated molecules in tissues and immune cells in health and in asthma, COPD, obesity, hypertension, and 
COVID-19 risk factors. Allergy 2020.

6. K KDD, S SKK, R RTT, et al. Coronavirus Disease 2019-COVID-19. Clin Microbiol Rev 2020; 33(4).

7. Raj VS, Mou H, Smits SL, et al. Dipeptidyl peptidase 4 is a functional receptor for the emerging human coronavirus-EMC. Nature,2013; 495(7440):251-254.

8. T TSS, K KOO, H HSS, et al. Polyarthropathy in type 2 diabetes patients treated with DPP4 inhibitors. Diabetes Res Clin Pract 2013;102(1):e8-e12.

9. L LFF, G GKK, M MRR. Are patients with hypertension and diabetes mellitus at increased risk for COVID-19 infection?. Lancet Respir Med 2020;8(4): e21.

10. Yang $X, Y u Y, X u$ J, et al. Clinical course and outcomes of critically ill patients with SARS-CoV-2 pneumonia in Wuhan, China: a single-centered, retrospective, observational study. The Lancet. Respiratory medicine 2020;8(5):475-481.

11. SB SBSS, A ADSDS, M MGG, et al. Dipeptidyl peptidase-4 (DPP4) inhibition in COVID-19. Acta Diabetol 2020;57(7):779-83.

12. Von Mering $C$, Huynen $M$, Jaeggi $D$, et al. STRING: a database of predicted functional associations between proteins. Nucleic Acids Res 2003;31:258-61.

13. Röhrborn D, Wronkowitz N, Eckel J. DPP4 in Diabetes. Frontiers in immunology 2015; 6:386.

14. Raha AA, Chakraborty S, Henderson J, et al. Investigation of CD26, a potential SARS-CoV-2 receptor, as a biomarker of age and pathology. Bioscience reports 2020; 40(12).

15. Ye Q, Wang B, Mao J. The pathogenesis and treatment of the 'Cytokine Storm' in COVID-19. The Journal of infection 2020; 80(6):607-13.

16. Wang J, Meng W. COVID-19 and diabetes: the contributions of hyperglycemia. Journal of molecular cell biology 2020;10(1):mjaa054.

17. McGonagle D, Sharif K, O'Regan A, et al. The Role of Cytokines including Interleukin-6 in COVID-19 induced Pneumonia and Macrophage Activation Syndrome-Like Disease. Autoimmunity reviews 2020;19(6):102537.

18. Shah FA, Mahmud H, Gallego-Martin T, et al. Therapeutic Effects of Endogenous Incretin Hormones and Exogenous Incretin-Based Medications in Sepsis. The Journal of clinical endocrinology and metabolism 2019; 104(11):5274-84.

19. Jang JH, Yamada Y, Janker F, et al. Anti-inflammatory effects on ischemia/reperfusion-injured lung transplants by the cluster of differentiation 26/dipeptidylpeptidase 4 (CD26/DPP4) inhibitor vildagliptin. The Journal of thoracic and cardiovascular surgery 2017; 153(3):713-24.e714.

20. Kokic Males V. Letter to the editor in response to the article "COVID-19 and diabetes: Can DPP4 inhibition play a role?". Diabetes research and clinical practice 2020;163:108163.

21. Scheen AJ, Marre M, Thivolet C. Prognostic factors in patients with diabetes hospitalized for COVID19: Findings from the CORONADO study and other recent reports. Diabetes \& metabolism 2020; 46(4):265-71. 
22. Chen Y, Yang D, Cheng B, et al. Clinical Characteristics and Outcomes of Patients With Diabetes and COVID-19 in Association With Glucose-Lowering Medication. Diabetes Care 2020; 43(7):1399-407.

23. Cariou B, Hadjadj S. Phenotypic characteristics and prognosis of inpatients with COVID-19 and diabetes: the CORONADO study. Diabetologia 2020;63(8):1500-15

24. Fadini GP, Morieri ML, Longato E, et al. Exposure to dipeptidyl-peptidase-4 inhibitors and COVID-19 among people with type 2 diabetes: A case-control study. Diabetes Obes Metab 2020; 22(10):194650

25. Kim MK, Jeon JH. The Clinical Characteristics and Outcomes of Patients with Moderate-to-Severe Coronavirus Disease 2019 Infection and Diabetes in Daegu, South Korea. Diabetes Metab J 2020;44(4):602-13

26. Mirani M, Favacchio G. Impact of Comorbidities and Glycemia at Admission and Dipeptidyl Peptidase 4 Inhibitors in Patients With Type 2 Diabetes With COVID-19: A Case Series From an Academic Hospital in Lombardy, Italy. Diabetes Care 2020;43(12):3042-9

27. Montastruc F, Romano C, Montastruc JL, et al. Pharmacological characteristics of patients infected with SARS-Cov-2 admitted to Intensive Care Unit in South of France. Therapie 2020; 75(4):381-4.

28. Solerte SB, D'Addio F, Trevisan R, et al. Sitagliptin Treatment at the Time of Hospitalization Was Associated With Reduced Mortality in Patients With Type 2 Diabetes and COVID-19: A Multicenter, Case-Control, Retrospective, Observational Study. Diabetes Care 2020; 43(12):2999-3006.

29. Zhang Y, Chua S, Jr. Leptin Function and Regulation. Comprehensive Physiology 2017; 8(1):351-69.

30. Wang J, Xu Y, Zhang $X$, et al. Leptin correlates with monocytes activation and severe condition in COVID-19 patients. Journal of leukocyte biology 2021;1(6):1-12.

31. Alberca RW, Oliveira LM, Branco A, et al. Obesity as a risk factor for COVID-19: an overview. Critical reviews in food science and nutrition 2020;1-15.

32. McElvaney OJ, McEvoy NL, McElvaney OF, et al. Characterization of the Inflammatory Response to Severe COVID-19 Illness. American journal of respiratory and critical care medicine 2020; 202(6):81221.

\section{Figures}




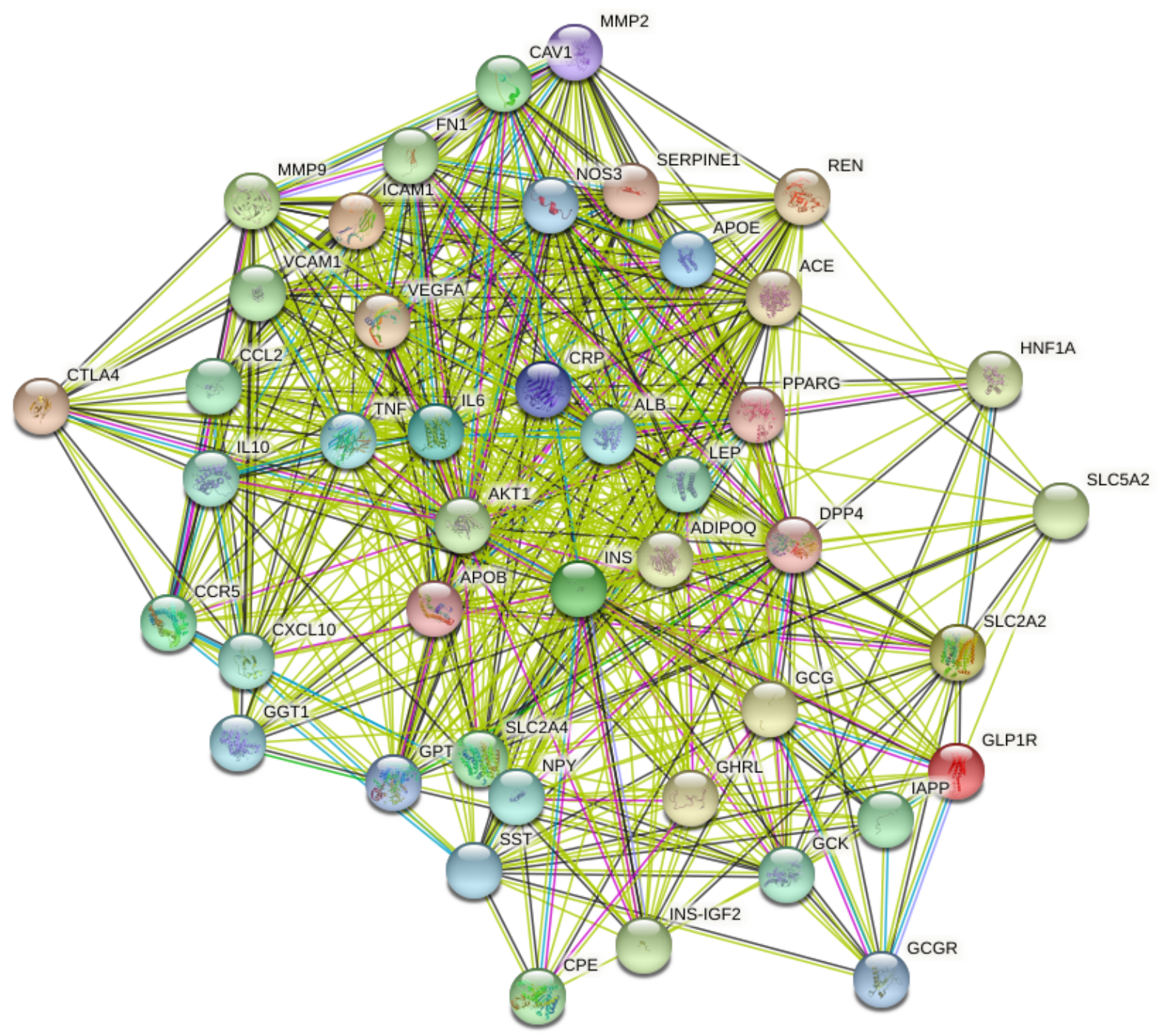

\section{Figure 1}

Interaction networ of DPP4 related protein 


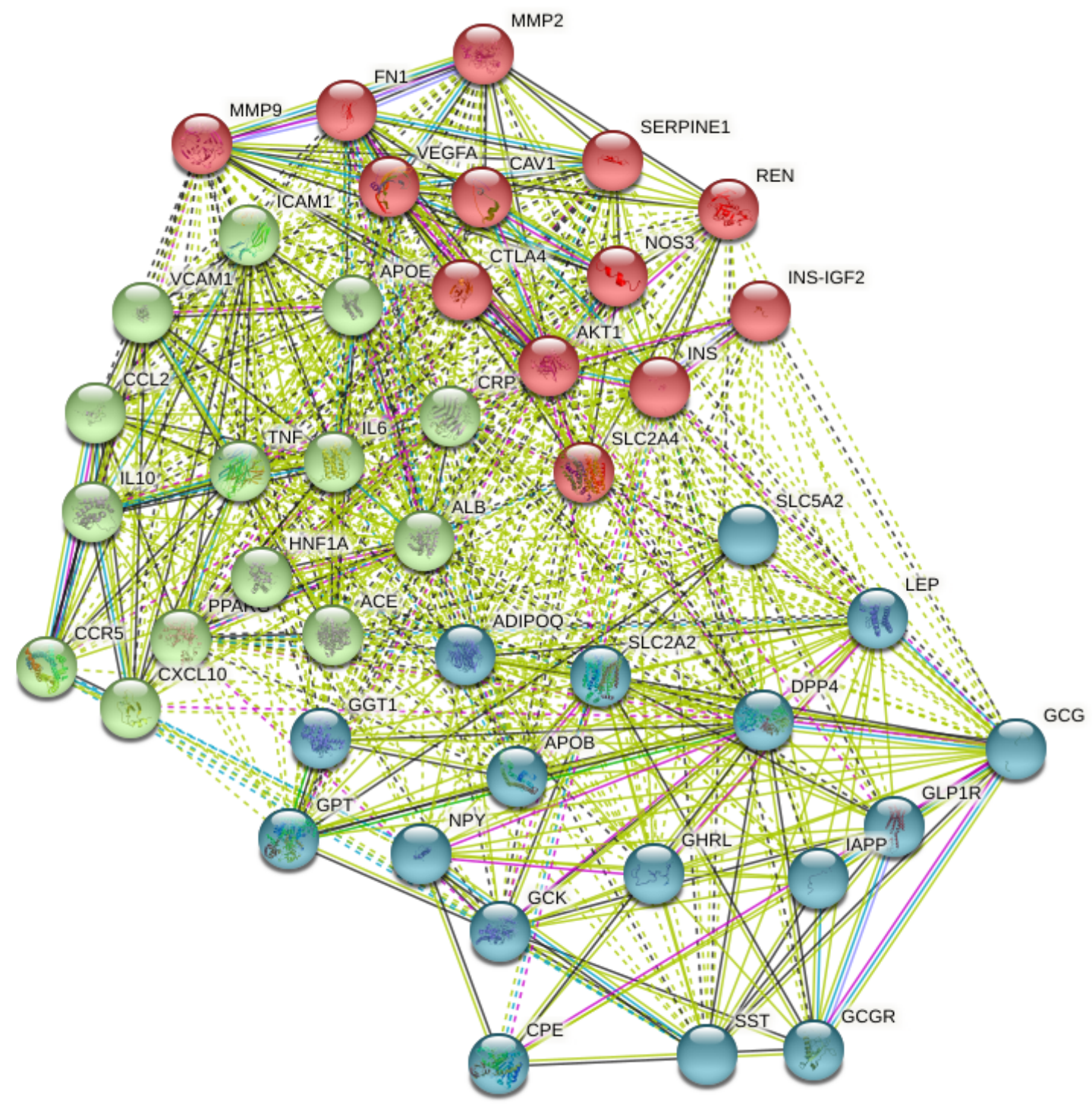

Figure 2

DPP4 related protein module recognition 